\title{
A Study on Solving the Seepage Flow Model of Three-Area Composite Reservoir
}

\author{
X.X. Dong \\ School of Mathematics and Computer Engineering \\ Xihua University \\ Chengdu, China \\ S.C. Li \\ School of Mathematics and Computer Engineering, \\ Xihua University \\ Chengdu, China
}

\author{
D.D. Gui \\ Beijing Dongrunke Petroleum Technology Co., Ltd. \\ Beijing, 100029, China
}

\author{
F.J. Zhang \\ Nexen Energy ULC \\ Calgary AB, T2P3P7, Canada
}

\begin{abstract}
This paper studies the seepage flow model of threearea composite reservoir under three kinds outer boundary conditions (infinite boundary, constant pressure boundary and closed outer boundary), in which influences of well-bore storage and effective radius are taken into consideration. On the basis of the theory of similar structure of the solution of the boundary value problem of differential equation, this paper obtains the solution of the seepage flow model of three-area composite reservoir. The study not only contributes to further analysis on the inherent law of the solution, but also supplements the study on composite reservoir.
\end{abstract}

Keywords-composite reservoir; seepage flow model; well-bore storage; effective radius; similar structure of solution; similar kernel function

\section{INTRODUCTION}

Composite reservoir is the reservoir that involves two regions with different properties (the rock-oriented one and the fluid-oriented one), namely, the inner region and the outer one are separated by a discontinuous interface.

[1] proposed a composite reservoir model of rock with fractal characteristics. Numerical solutions of the dynamic pressure of formation pressure are obtained by using the Laplace Numerical Inversion. [2] studied the equation of pressure diffusion in radically composite reservoirs by the application of Green function, Fourier transform and Laplace transform and given a general method to solve the class of equations. [3] established seepage flow mathematical model of multilayer two-area composite reservoir and solved the model. The study provides a theoretical basis for the multilayer reservoir thermal. [4-6] proposed mathematical model for well test analysis of perfectly describing the fractal composite reservoir under three kinds of outer boundary conditions. A similar structure of the solution was discovered by analyzing the percolation characteristics of fractal composite reservoir under three kinds of outer boundary conditions. By analyzing the solution of the reservoir pressure and dimensionless reservoir pressure distribution in Laplace space, [7] and [8] discovered the similar structure of the solution of the composite reservoir under the three kinds of outer boundary conditions. Furthermore, they made the theoretical graph and analyzed the impact of the well-bore storage and skin factor on dimensionless reservoir pressure and dimensionless bottom-hole pressure by using the numerical inversion. The percolation model of composite reservoir was established [9], which considers the effective well-bore radius and well-bore storage. According to the theory of similar structure, [9] defined similar kernel functions and obtained similar structures of solutions under three kings outer boundaries. Spherical fluid flow model for composite reservoir that introduces effective well-bore radius was established [10]. Seepage model was transformed into a boundary value problem of ordinary differential equation in Laplace space by using Laplace transform. The similar structure of the solution was obtained. Similar structures of solutions have the same expression in three kinds of outer boundary conditions.

However, above researches mainly studied two-area composite reservoir. On the basis of above researches, this paper establishes the seepage flow mathematical model of three-area composite reservoir under three kinds of outer boundary conditions (infinite boundary, constant pressure boundary and closed boundary), in which influences of wellbore storage and effective radius are taken into consideration. Then, on the basis of theory of similar structure of the solution of the boundary value problem of differential equation, the solution of the seepage flow mathematical model of three-area composite reservoir is obtained. The study further supplements the study on composite reservoir.

\section{THE SEEPAGE FLOW MODEL OF THREE-AREA COMPOSITE RESERVOIR}

This paper studies the model of three-area composite reservoir which influences of well-bore storage and effective radius are considered. The main assumptions of the model include as follows: law; 
2) Reservoir has equal thick, each direction is horizontal and has a same nature;

3) Neglecting the capillary single-phase horizontal flow without gravity effect; curve;

4) The well product at the constant rate and isothermal

5) There is no additional pressure drop at the interface of two seepage zone;

6) Formation pressure is initial reservoir pressure $p_{0}$ before producing.

Basic seepage equations of three-area composite reservoir which influences of well-bore storage and effective radius are considered are as follows:

Inner area:

$$
\frac{\partial^{2} p_{1}}{\partial r^{2}}+\frac{1}{r} \frac{\partial p_{1}}{\partial r}=\frac{1}{\eta_{1}} \frac{\partial p_{1}}{\partial t} \quad r_{\omega}<r<\alpha r_{\omega}, t>0
$$

Middle area:

$$
\frac{\partial^{2} p_{2}}{\partial r^{2}}+\frac{1}{r} \frac{\partial p_{2}}{\partial r}=\frac{1}{\eta_{2}} \frac{\partial p_{2}}{\partial t} \quad \alpha r_{\omega} \leq r \leq \beta r_{\omega}, t>0
$$

Outer area:

$$
\frac{\partial^{2} p_{3}}{\partial r^{2}}+\frac{1}{r} \frac{\partial p_{3}}{\partial r}=\frac{1}{\eta_{3}} \frac{\partial p_{3}}{\partial t} \quad r \geq \beta r_{\omega}, t>0
$$

Initial condition:

$$
p_{1}(r, 0)=p_{2}(r, 0)=p_{3}(r, 0)=p_{0} .
$$

Inner condition:

$$
\left\{\begin{array}{l}
p_{\omega}(t)=p_{1}\left(r_{\omega e}, t\right) \\
\left.\left(r \frac{\partial p_{1}}{\partial r}\right)\right|_{r=r_{\omega e}}=\frac{\mu_{1}}{2 \pi k_{1} h}\left(B q+C \frac{d p_{\omega}}{d t}\right) .
\end{array}\right.
$$

Convergence condition:

$$
\left\{\begin{array}{l}
p_{1}\left(\alpha r_{\omega}, t\right)=p_{2}\left(\alpha r_{\omega}, t\right) \\
\left.\frac{k_{1}}{\mu_{1}} \frac{\partial p_{1}}{\partial r}\right|_{r=\alpha r_{\omega}}=\left.\frac{k_{2}}{\mu_{2}} \frac{\partial p_{2}}{\partial r}\right|_{r=\alpha r_{\omega}} . \\
p_{2}\left(\beta r_{\omega}, t\right)=p_{3}\left(\beta r_{\omega}, t\right) \\
\left.\frac{k_{2}}{\mu_{2}} \frac{\partial p_{2}}{\partial r}\right|_{r=\beta r_{\omega}}=\left.\frac{k_{3}}{\mu_{3}} \frac{\partial p_{3}}{\partial r}\right|_{r=\beta r_{\omega}}
\end{array} .\right.
$$

Outer condition:

$$
\begin{cases}p_{3}(\infty, \mathrm{t})=p_{0} & \text { Infinite outer boundary condition } \\ p_{3 D}\left(\mathrm{R}_{D}, t_{D}\right)=0 & \text { Constant pressure outer boundary condition } \\ \left.\frac{\partial p_{3}}{\partial r}\right|_{r=R}=0 & \text { Closed outer boundary condition }\end{cases}
$$

III. SOlVING THE SEEPAGE FLOW MODEL OF THREE-AREA COMPOSITE RESERVOIR

In order to facilitate the research and description, firstly, dimensionless variables are introduced as follows:

$$
\begin{aligned}
& P_{i D}\left(r_{D}, t_{D}\right)=\frac{2 \pi k_{1} h}{B q \mu_{1}}\left[p_{0}-p_{i}(r, t)\right] \quad(i=1,2,3), \\
& \lambda_{1}=\left(\frac{k_{2}}{\mu_{2}}\right) /\left(\frac{k_{1}}{\mu_{1}}\right), t_{D}=\frac{k_{1} t}{\phi_{1} \mu_{1} C_{t_{1}} r_{\omega}^{2}}, \\
& C_{D}=\frac{C}{2 \pi \phi_{1} C_{t_{1}} h r_{\omega}^{2}}, r_{D}=\frac{r}{r_{\omega} e^{-S}}, T_{D}=\frac{t_{D}}{C_{D}}, \\
& \sigma_{2}=\frac{\eta_{2}}{\eta_{3}}=\frac{\phi_{3} \mu_{3} C_{t_{3}}}{\phi_{2} \mu_{2} C_{t_{2}}} \cdot \frac{k_{2}}{k_{3}}, R_{D}=\frac{R}{r_{\omega} e^{-S}}, \\
& \sigma_{1}=\frac{\eta_{1}}{\eta_{2}}=\frac{\phi_{2} \mu_{2} C_{t_{2}}}{\phi_{1} \mu_{1} C_{t_{1}}} \cdot \frac{k_{1}}{k_{2}} .
\end{aligned}
$$

Secondly, the Laplace transform is taken to the seepage flow model of three-area composite reservoir with dimensionless variable $t_{D}$, i.e.

$$
\begin{aligned}
& \bar{P}_{i D}\left(r_{D}, z\right)=\int_{0}^{\infty} e^{-z T_{D}} P_{i D}\left(r_{D}, T_{D}\right) d T_{D} \quad(i=1,2,3) \\
& \bar{P}_{\omega D}(z)=\int_{0}^{\infty} e^{-z T_{D}} P_{\omega D}\left(T_{D}\right) d T_{D} .
\end{aligned}
$$

Finally, the boundary value problem of the ODE with parameter $Z$ (where $Z$ is Laplace space variable) is obtained as below: 


$$
\left\{\begin{array}{l}
\frac{d^{2} \bar{P}_{1 D}}{d r_{D}^{2}}+\frac{1}{r_{D}} \cdot \frac{d \bar{P}_{1 D}}{d r_{D}}=\frac{z}{C_{D} e^{-2 S}} \bar{P}_{1 D}, \quad 1 \leq r_{D} \leq \alpha \\
\frac{d^{2} \bar{P}_{2 D}}{d r_{D}^{2}}+\frac{1}{r_{D}} \cdot \frac{d \bar{P}_{2 D}}{d r_{D}}=\frac{\sigma_{1} z}{C_{D} e^{-2 S}} \bar{P}_{2 D}, \quad \alpha \leq r_{D} \leq \beta \\
\frac{d^{2} \bar{P}_{3 D}}{d r_{D}^{2}}+\frac{1}{r_{D}} \cdot \frac{d \bar{P}_{3 D}}{d r_{D}}=\frac{\sigma_{2} z}{C_{D} e^{-2 S}} \bar{P}_{3 D}, \quad r_{D} \geq \beta \\
\bar{P}_{\omega D}(z)=\bar{P}_{1 D}(1, z) \\
\left(r_{D} \frac{d \bar{P}_{1 D}}{d r_{D}}\right)_{r_{D}=1}=-\bar{q}_{D}(z)+z \bar{P}_{\omega D} \quad \\
\bar{P}_{1 D}(\alpha, z)=\bar{P}_{2 D}(\alpha, z),\left.\quad \frac{d \bar{P}_{1 D}}{d r_{D}}\right|_{r_{D}=\alpha}=\left.\lambda_{1} \frac{d \bar{P}_{2 D}}{d r_{D}}\right|_{r_{D}=\alpha} \\
\bar{P}_{2 D}(\beta, z)=\bar{P}_{3 D}(\beta, z),\left.\quad \frac{d \bar{P}_{2 D}}{d r_{D}}\right|_{r_{D}=\beta}=\left.\lambda_{2} \frac{d \bar{P}_{3 D}}{d r_{D}}\right|_{r_{D}=\beta} \\
\bar{P}_{3 D}(\infty, z)=0 \text { or } \bar{P}_{3 D}\left(R_{D}, z\right)=0 \text { or }\left.\frac{d \bar{P}_{3 D}}{d r_{D}}\right|_{r_{D}=R_{D}}=0
\end{array}\right.
$$

On the basis of similar structure of the solution of the boundary value problem of ODE, solutions of each area of the boundary value problem (8) can be obtained respectively as follows:

$$
\bar{P}_{1 D}\left(r_{D}, z\right)=\bar{q}_{D}(z) \cdot \frac{1}{1+\frac{1}{-2+z \Phi_{1}(1, z)}} \cdot \frac{1}{-2+z \Phi_{1}(1, z)} \cdot \Phi_{1}\left(r_{D}, z\right) \quad\left(1 \leq r_{D} \leq \alpha\right)
$$

$$
\begin{aligned}
\bar{P}_{2 D}\left(r_{D}, z\right)=\bar{q}_{D}(z) \cdot \frac{1}{1+\frac{1}{-2+z \Phi_{1}(1, z)}} \cdot \frac{1}{-2+z \Phi_{1}(1, z)} \\
\cdot \frac{\Psi_{0,1}\left(\alpha, \alpha, \sqrt{\frac{z}{C_{D} e^{-2 S}}}\right)}{\Phi_{2}(\alpha, z) \sqrt{\frac{z}{C_{D} e^{-2 S}}} \Psi_{1,1}\left(1, \alpha, \sqrt{\frac{z}{C_{D} e^{-2 S}}}\right)+\lambda_{1} \Psi_{1,0}\left(1, \alpha, \sqrt{\frac{z}{C_{D} e^{-2 S}}}\right)} \cdot \Phi_{2}\left(r_{D}, z\right)\left(\alpha \leq r_{D} \leq \beta\right)
\end{aligned}
$$

$$
\begin{aligned}
& \bar{P}_{3 D}\left(r_{D}, z\right)=\bar{q}_{D}(z) \cdot \frac{1}{1+\frac{1}{-2+z \Phi_{1}(1, z)}} \cdot \frac{1}{-2+z \Phi_{1}(1, z)} \\
& \frac{\Psi_{0,1}\left(\alpha, \alpha, \sqrt{\frac{z}{C_{D} e^{-2 S}}}\right)}{\left[\Phi_{2}(\alpha, z) \sqrt{\frac{z}{C_{D} e^{-2 S}}} \Psi_{1,1}\left(1, \alpha, \sqrt{\frac{z}{C_{D} e^{-2 S}}}\right)+\lambda_{1} \Psi_{1,0}\left(1, \alpha, \sqrt{\frac{z}{C_{D} e^{-2 S}}}\right)\right]} \\
& \cdot \frac{\Psi_{0,1}\left(\beta, \beta, \sqrt{\sigma_{1} z}\right)}{\left[\Phi_{3}(\beta, z) \sqrt{\frac{\sigma_{1} z}{C_{D} e^{-2 S}}} \Psi_{1,1}\left(\alpha, \beta, \sqrt{\frac{\sigma_{1} z}{C_{D} e^{-2 S}}}\right)+\lambda_{2} \Psi_{1,0}\left(\alpha, \beta, \sqrt{\frac{\sigma_{1} z}{C_{D} e^{-2 S}}}\right)\right]} \cdot \Phi_{3}\left(r_{D}, z\right)\left(r_{D} \geq \beta\right)
\end{aligned}
$$

Where the similar kernel function of outer area is delimited as below:

$$
\Phi_{3}\left(r_{D}, z\right)=\Phi_{3 j}\left(r_{D}, z\right) \quad(j=1,2,3) .
$$

The similar kernel function of middle area is delimited as below:

$$
\Phi_{2}\left(r_{D}, z\right)=\Phi_{2 j}\left(r_{D}, z\right) \quad(j=1,2,3) .
$$

The similar kernel function of inner area is delimited as below:

$$
\Phi_{1}\left(r_{D}, z\right)=\Phi_{1 j}\left(r_{D}, z\right) \quad(j=1,2,3) .
$$

Where $j=1_{\text {denotes that the outer boundary condition is }}$ infinite, $j=2$ denotes that the outer boundary condition is constant pressure, $j=3$ denotes that the outer boundary condition is closed.

Case1. When the outer boundary condition is infinite $\bar{P}_{3 D}(\infty, z)=0$,

$$
\begin{aligned}
& \Phi_{31}\left(r_{D}, z\right)=-\frac{K_{0}\left(\sqrt{\frac{\sigma_{2} z}{C_{D} e^{-2 S}}} r_{D}\right)}{\sqrt{\frac{\sigma_{2} z}{C_{D} e^{-2 S}}} K_{1}\left(\beta \sqrt{\frac{\sigma_{2} z}{C_{D} e^{-2 S}}}\right)} \quad\left(\beta \leq r_{D} \leq \infty\right) \\
& \Phi_{21}\left(r_{D}, z\right)=\frac{\lambda_{2} \Psi_{0,0}\left(r_{D}, \beta, \sqrt{\frac{\sigma_{1} z}{C_{D} e^{-2 S}}}\right)+\sqrt{\frac{\sigma_{1} Z}{C_{D} e^{-2 S}}} \Psi_{0,1}\left(r_{D}, \beta, \sqrt{\frac{\sigma_{1} Z}{C_{D} e^{-2 S}}}\right) \Phi_{31}(\beta, z)}{\lambda_{2} \sqrt{\frac{\sigma_{1} Z}{C_{D} e^{-2 S}}} \Psi_{1,0}\left(\alpha, \beta, \sqrt{\sigma_{1} z}\right)+\frac{\sigma_{1} z}{C_{D} e^{-2 S}} \Psi_{1,1}\left(\alpha, \beta, \sqrt{\frac{\sigma_{1} Z}{C_{D} e^{-2 S}}}\right) \Phi_{31}(\beta, z)} \\
& \left(\alpha \leq r_{D} \leq \beta\right) \\
& \Phi_{11}\left(r_{D}, z\right)=\frac{\lambda_{1} \Psi_{0,0}\left(r_{D}, \alpha, \sqrt{\frac{z}{C_{D} e^{-2 S}}}\right)+\sqrt{\frac{z}{C_{D} e^{-2 S}}} \Psi_{0,1}\left(r_{D}, \alpha, \sqrt{\frac{z}{C_{D} e^{-2 S}}}\right) \Phi_{21}(\alpha, z)}{\lambda_{1} \sqrt{\frac{z}{C_{D} e^{-2 S}}} \Psi_{1,0}(1, \alpha, \sqrt{z})+\frac{z}{C_{D} e^{-2 S}} \Psi_{1,1}\left(1, \alpha, \sqrt{\frac{z}{C_{D} e^{-2 S}}}\right) \Phi_{21}(\alpha, z)} \\
& \left(1 \leq r_{D} \leq \alpha\right)
\end{aligned}
$$

Case2. When the outer boundary condition is constant pressure $^{\bar{P}_{3 D}}\left(R_{D}, z\right)=0$,

$$
\begin{gathered}
\Phi_{32}\left(r_{D}, z\right)=\frac{\Psi_{0,0}\left(r_{D}, R_{D}, \sqrt{\frac{\sigma_{2} Z}{C_{D} e^{-2 S}}}\right)}{\sqrt{\frac{\sigma_{2} Z}{C_{D} e^{-2 S}}} \Psi_{1,0}\left(\beta, R_{D}, \sqrt{\frac{\sigma_{2} Z}{C_{D} e^{-2 S}}}\right)} \quad\left(\beta \leq r_{D} \leq R_{D}\right) \\
\Phi_{22}\left(r_{D}, z\right)=\frac{\lambda_{2} \Psi_{0,0}\left(r_{D}, \beta, \sqrt{\frac{\sigma_{1} Z}{C_{D} e^{-2 S}}}\right)+\sqrt{\frac{\sigma_{1} Z}{C_{D} e^{-2 S}}} \Psi_{0,1}\left(r_{D}, \beta, \sqrt{\frac{\sigma_{1} z}{C_{D} e^{-2 S}}}\right) \Phi_{32}(\beta, z)}{\lambda_{2} \sqrt{\frac{\sigma_{1} Z}{C_{D} e^{-2 S}}} \Psi_{1,0}\left(\alpha, \beta, \sqrt{\frac{\sigma_{1} Z}{C_{D} e^{-2 S}}}\right)+\frac{\sigma_{1} Z}{C_{D} e^{-2 S}} \Psi_{1,1}\left(\alpha, \beta, \sqrt{\frac{\sigma_{1} z}{C_{D} e^{-2 S}}}\right) \Phi_{32}(\beta, z)} \\
\left(\alpha \leq r_{D} \leq \beta\right)
\end{gathered}
$$

$$
\begin{gathered}
\Phi_{12}\left(r_{D}, z\right)=\frac{\lambda_{1} \Psi_{0,0}\left(r_{D}, \alpha, \sqrt{\frac{z}{C_{D} e^{-2 S}}}\right)+\sqrt{\frac{z}{C_{D} e^{-2 S}}} \Psi_{0,1}\left(r_{D}, \alpha, \sqrt{\frac{z}{C_{D} e^{-2 S}}}\right) \Phi_{22}(\alpha, z)}{\lambda_{1} \sqrt{\frac{z}{C_{D} e^{-2 S}}} \Psi_{1,0}\left(1, \alpha, \sqrt{\frac{z}{C_{D} e^{-2 S}}}\right)+\frac{z}{C_{D} e^{-2 S}} \Psi_{1,1}\left(1, \alpha, \sqrt{\frac{z}{C_{D} e^{-2 S}}}\right) \Phi_{22}(\alpha, z)} \\
\left(1 \leq r_{D} \leq \alpha\right)
\end{gathered} .
$$

Case3. When the outer boundary condition is closed $\left.\frac{d \bar{P}_{3 D}}{d r_{D}}\right|_{r_{D}=R_{D}}=0$, 


$$
\begin{gathered}
\Phi_{33}\left(r_{D}, z\right)=\frac{\Psi_{0,1}\left(r_{D}, R_{D}, \sqrt{\frac{\sigma_{2} Z}{C_{D} e^{-2 S}}}\right)}{\sqrt{\frac{\sigma_{2} Z}{C_{D} e^{-2 S}}} \Psi_{1,1}\left(\beta, R_{D}, \sqrt{\frac{\sigma_{2} Z}{C_{D} e^{-2 S}}}\right)} \quad\left(\beta \leq r_{D} \leq R_{D}\right) \\
\Phi_{23}\left(r_{D}, z\right)=\frac{\lambda_{2} \Psi_{0,0}\left(r_{D}, \beta, \sqrt{\frac{\sigma_{1} Z}{C_{D} e^{-2 S}}}\right)+\sqrt{\frac{\sigma_{1} Z}{C_{D} e^{-2 S}}} \Psi_{0,1}\left(r_{D}, \beta, \sqrt{\frac{\sigma_{1} Z}{C_{D} e^{-2 S}}}\right) \Phi_{33}(\beta, z)}{\lambda_{2} \sqrt{\frac{\sigma_{1} Z}{C_{D} e^{-2 S}}} \Psi_{1,0}\left(\alpha, \beta, \sqrt{\frac{\sigma_{1} Z}{C_{D} e^{-2 S}}}\right)+\frac{\sigma_{1} Z}{C_{D} e^{-2 S}} \Psi_{1,1}\left(\alpha, \beta, \sqrt{\frac{\sigma_{1} Z}{C_{D} e^{-2 S}}}\right) \Phi_{33}(\beta, z)} \\
\left(\alpha \leq r_{D} \leq \beta\right)
\end{gathered}
$$

$$
\begin{gathered}
\Phi_{13}\left(r_{D}, z\right)=\frac{\lambda_{1} \Psi_{0,0}\left(r_{D}, \alpha, \sqrt{\frac{z}{C_{D} e^{-2 S}}}\right)+\sqrt{\frac{z}{C_{D} e^{-2 S}}} \Psi_{0,1}\left(r_{D}, \alpha, \sqrt{\frac{z}{C_{D} e^{-2 S}}}\right) \Phi_{23}(\alpha, z)}{\lambda_{1} \sqrt{\frac{z}{C_{D} e^{-2 S}}} \Psi_{1,0}\left(1, \alpha, \sqrt{\frac{z}{C_{D} e^{-2 S}}}\right)+\frac{z}{C_{D} e^{-2 S}} \Psi_{1,1}\left(1, \alpha, \sqrt{\frac{z}{C_{D} e^{-2 S}}}\right) \Phi_{23}(\alpha, z)} \\
\left(1 \leq r_{D} \leq \alpha\right)
\end{gathered}
$$

\section{Where}

$$
\Psi_{m, n}(\alpha, \beta, y)=I_{m}(\alpha y) K_{n}(\beta y)+(-1)^{m-n+1} K_{m}(\alpha y) I_{n}(\beta y) \text { and }
$$

$I_{n}(\cdot), K_{n}(\cdot)$ are respectively the first and the second class of modified Bessel functions of order $n_{[11]}$.

Let $r_{D}=1$ and substitute Eq.(9) into Eq.(8.4), we can obtain the solution of dimensionless bottom-hole pressure in Laplace space:

$$
\bar{P}_{\omega D}(z)=\bar{q}_{D}(z) \cdot \frac{1}{1+\frac{1}{-2+\Phi_{1}(1, z)}} \cdot \frac{1}{-2+\Phi_{1}(1, z)} \cdot \Phi_{1}(1, z)
$$

\section{CONCLUSIONS}

Based on the reasoning mentioned above, the following conclusions can be drawn.

1) Base on the theory of similar structure of the solution of the boundary value problem of differential equation, this paper easily obtains the solution of the seepage flow model of three-area composite reservoir. And the solution has the form of the product of continued fraction.

2) Base on the continued fraction Eqs.(9)-(11), we can directly analyze the connection between solutions of inner, middle and outer areas and two linear independent solutions of governing equations, functions of guide solution and similar kernel functions.

3) The seepage flow model of three-area composite reservoir has a same similar structure of solutions under three different outer boundary conditions. The difference is that similar kernel functions are different under three different outer boundary conditions.

\section{NOMENCLATURE}

$B$ - Formation volume factor, dimension; $C_{t}$ Compressibility, $\mathrm{MPa}^{-1}$; $\mathrm{C}$ —Well-bore storage coefficient, $m^{3} / \mathrm{MPa} ; k_{i}$ _Permeability, $\mu m^{2} ; p_{i}$-Reservoir pressure, MPa ; $p_{\omega}$ _Bottom-hole pressure , MPa; $q$ _-Production rate or injection rate, $\mathrm{m}^{3} / \mathrm{d} ; \phi_{i}$ - Porosity, dimensionless; $\mathrm{R}$ — The outer boundary radius, $m ; r$ Well-bore radius, $m$; $S$ _Skin factor, dimensionless; $\mu_{i}$ Viscosity, $M P a \cdot s ; P_{0}$ _Initial reservoir pressure, $M P a$; $h$ - Reservoir thickness, $m$; $z$ _Laplace space variable; $\eta_{i}$ _Pressure transmitting coefficient, $\mu \mathrm{m}^{2} \cdot \mathrm{MPa} ; t$ Time.

\section{ACKNOWLEDGMENT}

This Project is supported by the Science and Technology Innovation Project "Xihua Cup" of Xihua University under Grant No. 2014039 and the Scientific Research Fund of Sichuan Provincial Education Department of China under Grant No.12ZA164.

\section{REFERENCES}

[1] Poon D. innernational Meeting of the Pet.Soc. of. CIM/SPE9-534, 1995.

[2] Fikri J, Kuchuk, Tarek M, Habashy. Solution of Pressure Diffusion in Radially Composite Reservoirs [J]. Transport in Porous Media. 19(1995): 199-232.

[3] Abdurrahman Satman. An Analytical Study of Transient Flow in Stratified Systems with Fluid Banks[A. SPE 10264, 1997.

[4] S.C. Li, T.P. Zheng. A model solution of testing analysis in fractal composite reservoir (in Chinese) [J]. Journal of Jilin University (Engineering and Technology Edition), 2004, 34:104-107.

[5] X.R. Deng , S.C. Li. Solution to the Well Testing Model for Fractal Composite Reservoirs (in Chinese) [J]. Journal of Xihua University (Natural Science Edition), 2005, 24(2):4-7.

[6] C.X. Xu, S.C. Li, W.B. Zhu. The Similar Structure of Pressure Distribution in the Fractal Composite Reservoir (in Chinese)[J]. Drilling \& Production Technology, 2006, 29(5):39-42.

[7] P.S. Zheng, S.C. Li, W.Z. Xu. WELL ANALYSIS METHOD BASED ON THE SIMILAR STRUCTURE OF PRESSURE DISTRIBUTION IN THE COMPOSITE RESERVOIR (in Chinese) [J]. Drilling \& Production Technology, 2007, 30(3): 49-50(-62).

[8] S.C. Li, P.S. Zheng, Y.F. Zheng. The Similar Structure of Pressure Distribution in the Composite Reservoir (in Chinese) [J]. Journal of Mathematics in Practice and Theory, 2008, 38(3):23-28.

[9] Q.Y. Li, S.C. Li, W. Li, Y.B. Tang. Study on percolation model of composite media reservoir based on similar structure of solution (in Chinese) [J]. Fault-Block Oil \& Gas Field, 2011, 18(5): 623-625.

[10] J.C. Wang, S.C. Li, L. Xu. Spherical Fluids Flow Models in Composite Reservoir Based on Similar Structure of Solution (in Chinese) [J]. Journal of Guilin University of Technology, 2012, 32(4) 624-627.

[11] S.S. Liu, S.D. Liu. Special Function [M]. Beijing: China Meteorological Press, 2002. 